\title{
Erratum to: Pedobacter bambusae sp. nov., isolated from soil of a bamboo plantation
}

\author{
Kyung Hwa Won • MooChang Kook • \\ Tae-Hoo Yi
}

Published online: 23 January 2015

(C) Springer International Publishing Switzerland 2015

Erratum to: Antonie van Leeuwenhoek (2015)

107:565-573

DOI: 10.1007/s10482-014-0352-8

The original article has been published incorrectly with an error in the affiliation. The correct version is given in this erratum.

The online version of the original article can be found under doi:10.1007/s10482-014-0352-8.

K. H. Won · T.-H. Yi ( $₫)$

Department of Oriental Medicinal Material and Processing, College of Life Science, Kyung Hee University, Yongin, Gyeonggi 446-701, Republic of

Korea

e-mail: drhoo@khu.ac.kr

M. Kook

Department of Marine Biotechnology, Anyang

University, Incheon 417-833, Republic of Korea 